\title{
Practical Applications of Modeling To Be Highlighted at Superalloys 2012
}

\section{Eric Huron}

Superalloys are on the verge of yet another revolutionary step in their development through the application of modeling and simulation. The industries that rely on superalloys most heavily are pushing the boundaries of materials performance in their quest for greater efficiencies and more cost-effective options. To address these challenges effectively, each superalloy must be closely tailored to its particular application with minimal development time and carefully optimized properties. This requires even greater understanding of the physical factors driving properties, using both extensive empirical relationship databases to guide the optimization strategies, as well as the development of fundamental models to fully address risks, such as stability and environmental interactions.

Modeling and simulation are critical to mining the existing data for true understanding of alloy and process impacts on performance, to guiding further refinement in composition and process, to shortening development times by eliminating testing and repetitive validation trials, and to more rapidly addressing production and field usage issues.

The practical application of modeling and simulation will be a particular focus at the 12th International Symposium on Superalloys, scheduled for September 9-13, 2012 at the Seven Springs Mountain Resort in Champion, Pennsylvania. Held every four years over a span reaching four decades, the symposium is considered the premier forum for the latest and most significant innovations in superalloys.

A particularly valuable aspect of the symposium is the refereed publication of all papers presented, resulting in an im- portant reference library for every superalloy metallurgist. Looking back through these proceedings provides an impressive chronology of superalloy development. Blade alloys have progressed through five generations; powder metallurgy disk alloys have progressed through four generations; disk processing has moved from relatively simple hot isostatic pressing to extensively modeled thermal mechanical processing via isothermal forging; and coatings have evolved from simple monolayers to multi-component systems.

The Superalloys 2012 symposium will continue exploring these traditional areas of alloy development, processing, coatings and environmental effects, and mechanical behavior, while incorporating a new emphasis on how applications in modeling and simulation are enabling advances. For instance, forge and heat treat process modeling has been the subject of several papers over successive conferences and further reporting is expected at Superalloys 2012. The evolution of alloy development modeling - from extensive trial-and-error broad experimentation to fundamentally based composition design - has also been traced from conference to conference and will be a key topic for 2012. Microstructural evolution models have likewise emerged in more recent conferences, with the upcoming meeting exploring the growing sophistication in the fidelity and predictive capability of these models. Finally, conference topics will examine how sophisticated, fundamentally based, three-dimensional models are being developed to predict defects, dendritic structures, solidification rates, and chemical segregation.

Bill Gostic, vice president of Advanced Programs and Technology, Pratt
\& Whitney (P\&W), will open Superalloys 2012 with a keynote address that reviews the broader application of modeling to the design and development of the Joint Strike Fighter engine and other $\mathrm{P} \& \mathrm{~W}$ advanced engines. He will also discuss the contributions of materials engineers toward the development of advanced gas turbine engines.

As always, the conference will be dedicated to an honoree who has made sustained contributions to the superalloy field. Looking back, the honorees to date represent a list of pioneers who have driven many advances mentioned in this article, including Carl Lund, John Radavich, Fred Pettit, Wilfred "Red" Couts, and Raymond Decker.

Superalloys 2012 will offer a traditional mix of morning and evening presentations with afternoons available for guests to network and catch up on the latest news from colleagues in the superalloys industry. The conference format will build on the success of the interactive session approach introduced in 2008, with multiple sessions scheduled so that attendees have a number of opportunities to participate and engage in deeper technical discussions with an array of presenters over various sessions. The attendees should find the interactive papers to be just as key and innovative as those presented in the oral sessions.

Extended abstracts for Superalloys 2012 are being accepted until September 1 . Authors can submit their abstracts via ProgramMaster at www.tms.org /Meetings/Specialty/Superalloys2012/ home.aspx.

Eric Huron is manager of Composites Technology at GE Aviation and is Program Committee chair for the 12th International Superalloys Committee.

\section{Eric Huron is a TMS Member!}

To read more about him, turn to page 10. To join TMS, visit www.tms.org/Society/Membership.aspx. 